\title{
DISTONIAS
}

\section{ASPECTOS CLÍNICOS E TERAPÊUTICOS EM 64 PACIENTES}

\section{JAMES PITÁGORAS DE MATTOS *, ANA LÚCIA ZUMA DE ROSSO* *, SÉRGIO NOVIS**}

\begin{abstract}
RESUMO - Os Autores apresentam a experiência em 64 pacientes, com as várias formas clínicas de apresentação de distonias, acompanhados no Setor de Doenças Extrapiramidais do Serviço de Neurologia do Hospital Universitário Clementino Fraga Filho da UFRJ, assim como, revisam a literatura, cotejando os resultados. $\mathrm{O}$ acompanhamento desses pacientes durante 5 anos e 6 meses resultou nas seguintes observações: 33 do sexo masculino e 31 do feminino; 48 brancos, 10 pardos e 6 negros; a média do tempo de doença foi 9 anos e 8 meses. Quanto à distribuição do movimento anormal, 30 (46,9\%) eram focais; $17(26,6 \%)$, segmentares; 13 (20,3\%), generalizadas; $3(4,7 \%)$, hemidistonias; 1 (1,5\%), multifocal. Quanto à idade de início, em $11(17,2 \%)$ se apresentou antes dos 12 anos; em $6(9,4 \%)$, entre 13 e 20 anos; e em $47(73,4 \%)$, após os 20 anos. Correspondiam à origem idiopática esporádica, $39(60,9 \%)$; idiopática familiar, $6(9,4 \%)$; sintomática, $19(29,7 \%)$. No que se refere à abordagem terapêutica destes pacientes, destacamos o emprego de anticolinérgicos, de agonistas e antagonistas dopaminérgicos e do baclofen isolado ou associado aos anticolinérgicos para as formas generalizadas. Para as distonias focais, os Autores concluem ser a toxina botulínica do tipo A o agente terapêtico mais eficaz aconselhado atualmente.
\end{abstract}

PALAVRAS-CHAVE: distonia, distonia musculorum deformans, toxina botulínica

\section{Dystonias: clinical and therapeutic features in 64 patients}

ABSTRACT - The experience with 64 patients with dystonia seen at the Extrapyramidal Diseases Sector of the Neurology Department of the Hospital Universitário Clementino Fraga Filho of the UFRJ is presented as well as the pertinent review of the literature. The five-and-a-half-year of follow-up showed that 33 were male and 31 female; 48 were white, 10 mulatto and 6 negro; the mean time of disease was 9 years and 8 months. According to the distribution of the movement disorder, $30(46.9 \%)$ were focal, $17(26.6 \%)$ segmental, $13(20.3 \%)$ generalized, $3(4.7 \%)$ hemidystonia and $1(1.5 \%)$ multifocal. In $11(17.2 \%)$ the age of onset was before 12 years old, in $6(9.4 \%)$ between 13 and 20 years old, and in $47(73.4 \%)$ after 20 years old. According to the etiology, 39 $(60.9 \%)$ were idiopathic sporadic, $6(9.4 \%)$ were idiopathic familial and $19(29.7 \%)$ were symptomatic. The therapeutical approach used in these patients were anticholinergic drugs, dopaminergic agonists or antagonists and baclofen, alone or associated with anticholinergic drugs for generalized dystonia. The authors conclude that botulinum toxin type $\mathrm{A}$ is the most valuable therapeutic agent in the treatment of focal dystonia.

KEY-WORDS: dystonia, dystonia musculorum deformans, botulinum toxin.

Schwalbe", em 1908, defendeu sua tese de doutorado "Chronic cramp syndrome with hysterical symptoms", a propósito de três pacientes de uma mesma família. Oppenheim ${ }^{19}$, três anos após (1911), descreveu seis casos novos, discordando da origem psicológica e defendendo a natureza orgânica dos sintomas. Na ocasião, Oppenheim empregou dois diferentes termos para a condição: Dysbasia Lordotica Progressiva (para enfatizar o caráter progressivo) e Dystonia Musculorum Deformans

Estudo realizado no Serviço de Neurologia do Hospital Universitário Clementino Fraga Filho da Universidade Federal do Rio de Janeiro (UFRJ): * Professor Adjunto e Chefe do Setor de Doenças Extrapiramidais; ** Médica; *** Professor Titular e Chefe de Serviço. Aceite: 26-outubro-1995. 
(para destacar as deformidades) ${ }^{1}$. Nesse mesmo ano, Flatau e Sterling (apud Fahn e col. ${ }^{7}$ ), descreveram mais dois casos, em meninos, com a doença, enfatizando a natureza orgânica, assim como, a provável origem hereditária. Ainda, em 1911, Oppenheim (apud Fahn e col. ${ }^{7}$ ) introduziu e definiu o termo distonia. Originalmente descreveu que "havia um estado de hipotonia em uma ocasião e de hipertonia em outra, geralmente, mas não necessariamente, desencadeado por movimentos voluntários". Excetuando-se o fato de ter descrito a coexistência de hipotonia e de hipertonia, também anotara posturas em torçāo afetando os membros e o tronco, a marcha bizarra, movimentos em abalos rápidos e, às vezes, rítmicos, a evoluçāo progressiva e as deformidades. Antes e depois da introdução do termo distonia, vários autores descreveram, clinicamente, o movimento anormal, destacando-se, entre outros, Gowers, Schwalbe, Oppenheim, Flatau e Sterling, Herz, Denny-Brown, Fahn, Marsden e Obeso (apud Fahn e col. ${ }^{7}$ ). Em 1984, o Comitê da Scientific Advisory Board of the Dystonia Medical Reseanch Foundation definiu distonia da seguinte forma: síndrome caracterizada por contrações musculares mantidas, frequentemente causando torções e movimentos repetitivos ou posturas anormais ${ }^{7}$. Atualmente, são classificadas em três formas, dependendo da etiologia, da idade do início e da distribuição dos movimentos anormais. Sob o ponto de vista etiológico, podem ser de dois tipos: idiopática (ou primária) e sintomática (ou secundária). A idiopática pode ser familiar ou esporádica. A familiar pode ter padrões de transmissão autossômica dominante, autossômica recessiva ou ligada ao sexo. As sintomáticas incidem em, aproximadamente, $30 \%$ dos casos e correspondem àquelas associadas a doenças ncurológicas hereditárias, a causas ambientais e a parkinsonismo e distonia psicogênica. A classificação quanto à idade de início é muito importante por causa do prognóstico. Quanto mais cedo começar a distonia, pior será o prognóstico, isto é, há tendência em se tornar mais grave e em se generalizar. Podem ser de início na infância (até 12 anos), na adolescência (13-20 anos) e na idade adulta (após os 20 anos). Quanto à distribuição, podem ser focal, segmentar, unilateral (hemidistonia),multifocal e generalizada. Na focal, há o envolvimento de uma única regiāo, sendo exemplos, pálpebra (blefaroespasmo), boca (distonia oromandibular), laringe (disfonia espástica), pescoço (torcicolo), membro superior (cãibra profissional). Na segmentar, há ocorrência de duas ou mais regiões contíguas, como, por exemplo, crânio-cervical (síndrome de Meige). Na hemidistonia, há o acometimento de toda uma metade corporal. Na multifocal, de duas ou mais regiōes nāo-contíguas, como, pescoço e membro inferior. Na generalizada, há a combinação de distonia crural segmentar com qualquer outro segmento ${ }^{5,7}$.

No início de 1990, o Serviço de Neurologia do Hospital Universitário Clementino Fraga Filho (HUCFF) da UFRJ criou o Setor de Doenças Extrapiramidais constituído, basicamente, para a assistência especializada aos pacientes em regime ambulatorial e de enfermaria, assim como para servir às atividades de ensino e pesquisa. Desde então, temos acompanhado o crescimento desse setor, contando, atualmente (junho-1995), com mais de 500 pacientes protocolados, 64 desses com as mais variadas formas de distribuição de distonias. Passaremos a discutir seus aspectos clínicos e terapêuticos.

\section{MATERIAL E MÉTODOS}

Constam do nosso material os pacientes acompanhados no Setor de Doenças Extrapiramidais do Serviço de Neurologia do HUCFF-UFRJ, em sua maioria, assim como, de outros, provenientes da clínica particular. A maioria refere-se a população adulta, pois as crianças são, habitualmente, avaliadas e acompanhadas no Instituto de Pediatria Martagão Gesteira, anexo ao nosso Hospital. Eventualmente, casos especiais são encaminhados ao nosso setor. Todos os pacientes foram diagnosticados baseados em elementos clínicos (critérios de inclusão), seguindo-se a definição de distonia do Comitê da Scientific Advisory Board of the Dystonia Medical Research Foundation'. Excluimos os casos recentemente protocolados, com exames complementares em andamento. Exames de sangue, de urina, eletrofisiológicos (EEG e ENMG) e de neuro-imagem (TC e RM de crânio) foram efetuados na maioria dos pacientes.

\section{RESULTADOS}

O acompanhamento de 64 pacientes distônicos, no período de janeiro-1990 a junho-1995, resultou nas seguintes observaçōes: a média de idade, por ocasião da última consulta, foi 45,5 anos 
(8 anos para o mais jovem e 81 anos para o mais idoso); 33 eram do sexo masculino e 31 do feminino; quanto ao grupo étnico, 48 eram brancos, 10 pardos e 6 negros; a média do tempo de acompanhamento foi 2 anos e 3 meses, e a média do tempo de doença, 9 anos e 8 meses.

No que se refere à distribuição do movimento anormal, em 30 pacientes $(46,9 \%)$ era focal; em $17(26,6 \%)$, segmentar; em $13(20,3 \%)$, generalizada; em $3(4,7 \%)$, hemidistonia; e em $1(1,5 \%)$, mutifocal (Fig 1). Dentre as focais, 11 tinham blefaroespasmo; 9, torcicolo; 9, na mão (sendo 6 específicas da escrita e 3 não-específicas); e 1, do pé. Dos 17 pacientes com distonia segmentar, 9 apresentavam a síndrome de Meige, 3 eram braquiais, 3 cérvico-braquiais e 2 crânio-cervicais. Dos 3 com hemidistonia, 2 localizavam-se à direita e 1 à esquerda. $O$ único paciente com distonia multifocal apresentava a sintomatologia na mão esquerda e no pé direito.

Quanto à idade de início, em $11(17,2 \%)$ a distonia começou antes dos 12 anos; em $6(9,4 \%)$, entre 13 e 20 anos; e em $47(73,4 \%$ ), após os 20 anos (Fig 2). Em relação à etiologia do processo, em 39 pacientes $(60,9 \%)$ era idiopática esporádica; em $6(9,4 \%)$, idiopática familiar e em $19(29,7 \%)$, sintomática (Fig 3). Dentre as sintomáticas, 8 casos eram por uso de neurolépticos, 4 correspondiam à forma hipercinética da encefalopatia crônica da infância, 3 de origem psicogênica, 2 vasculares (infarto cerebral), 1 com doença de Wilson e 1 associado a cisto aracnóideo supra-selar.

\section{DISCUSSÃO}

A ocorrência, praticamente igual, tanto no sexo masculino quanto no feminino, está de acordo com o encontrado na literatura ${ }^{2,7,17}$; no entanto, em nosso meio, Andrade e Ferraz ${ }^{2}$, analisando 76 pacientes com distonia idiopática, encontraram maior incidência no feminino. Quanto à distribuição das distonias, também como mostra a literatura ${ }^{7,17}$, quase a metade $(46,9 \%)$ era focal $(\mathrm{Fig} 1)$. $\mathrm{O}$ tipo mais comum de distonia focal observado foi o blefaroespasmo (11), logo seguido do torcicolo (9). No que se refere à idade do início do movimento anormal, a maioria $(73,4 \%)$ começou após os 20 anos (Fig 2), ressaltando que o nosso Hospital atende, basicamente, a pacientes adultos.

Como na literatura consultada $7,9,29,7 \%$ dos nossos pacientes pertencia ao grupo sintomático, no que se refere à etiologia, sendo a maioria por uso de neurolépticos ou como forma hipercinética da encefalopatia crônica da infância, resultados similares aos relatados, em nosso meio, por Ferraz

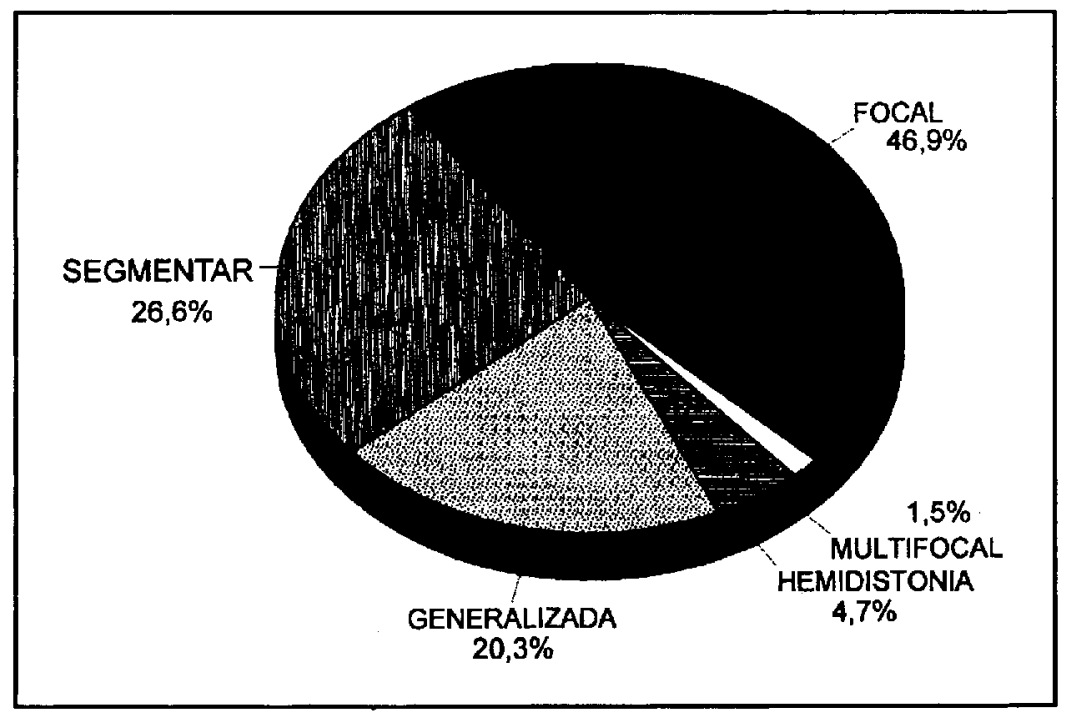

Fig. 1. Resultados quanto à distribuiçāo das distonias em 64 pacientes. 


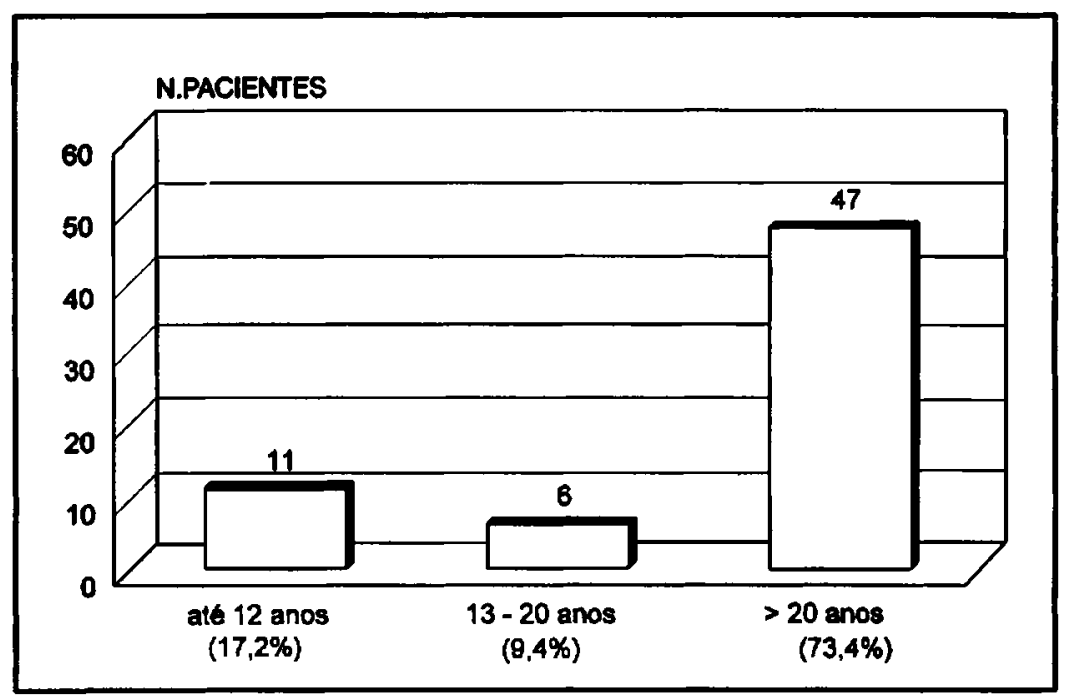

Fig. 2. Resultados quanto à idade de início das distonias em 64 pacientes.

e Andrade, a propósito de 46 pacientes $^{9}$. Ressalta-se que $3(4,7 \%)$ dos nossos pacientes eram sugestivos de serem de natureza psicogênica, de acordo com os critérios estabelecidos por Fahn ${ }^{6}$ e por Fahn e Williams $^{8}$. Do grupo idiopático, $60,9 \%$ era esporádico e somente $9,4 \%$, familiar. Este dado corresponde, aproximadamente, à metade do que registraram Marsden e col. ${ }^{17}$, provavelmente pelo fato de os pacientes com idade abaixo de 13 anos serem atendidos em outro Departamento (Fig 3).

$\mathrm{O}$ tratamento farmacológico das distonias pode ser dividido em medidas medicamentosas específicas (ou etiológicas) e não específicas (sintomáticas) ${ }^{7}$.

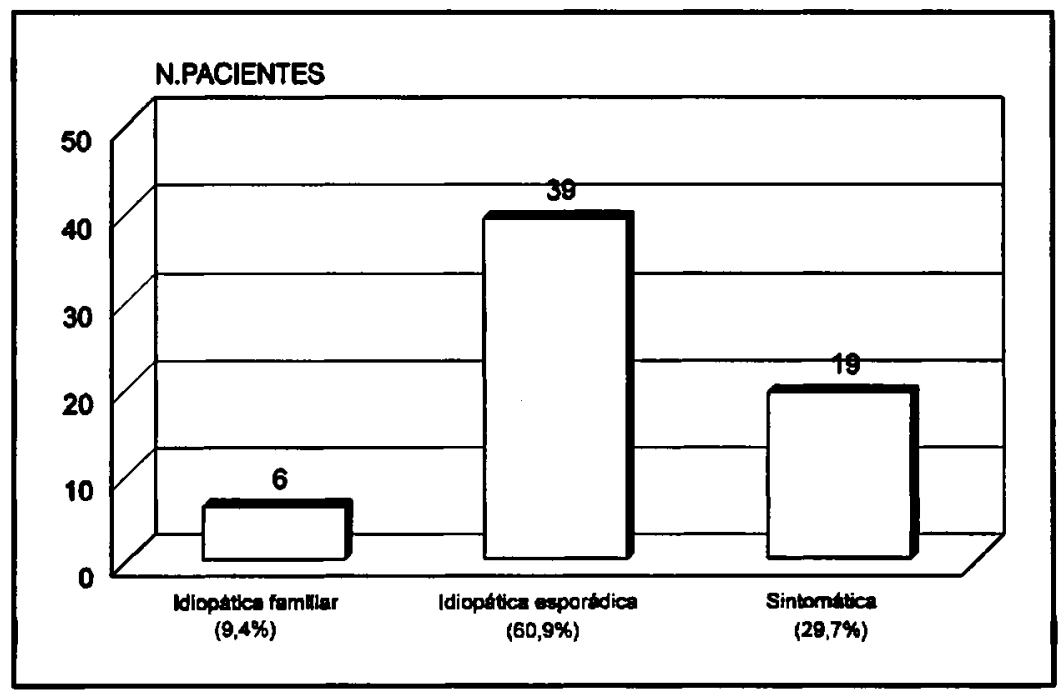

Fig. 3. Resultados quanto à etiologia das distonias em 64 pacientes. 


\section{A) Tratamento farmacológico específico}

A doença de Wilson (degeneração hepato-lenticular) é um excelente exemplo de como se tratar, especificamente. Afora a orientação dietética, aconselha-se o emprego de quelantes do cobre como a penicilamina-D e, modernamente, o sulfato de zinco. As distonias agudas induzidas por drogas, tais como, anticonvulsivantes, neurolépticos, metoclopramida e levodopa. Nas determinadas por uso de anticonvulsivantes, basta reduzir as doses das mesmas; nas induzidas por neurolépticos e por metoclopramida, administrar anticolinérgicos ou anti-histamínicos, por via parenteral; nas induzidas pela levodopa (de pico de dose), reduzindo-se a dose desta; a distonia matinal, aumentandose a última dose de levodopa ou utilizando-se a apresentação de ação prolongada. A distonia tardia é muito difícil de ser controlada, podendo desaparecer com a suspensāo do neuroléptico. Drogas antidopaminérgicas como, por exemplo, a tetrabenazina, são eficazes em $50 \%$ dos casos e os anticolinérgicos, como por exemplo o tri-hexifenidil e o biperideno, em $40 \%$ ?

\section{B) Tratamento farmacológico inespecifico}

Pode ser local ou sistêmico. O local consta do emprego da toxina botulínica; o sistêmico inclui o emprego de anticolinérgicos, de agonistas e de antagonistas dopaminérgicos, assim como de outros agentes?

Desde a introdução da toxina botulínica do tipo A para o tratamento do estrabismo, feita por Scott ${ }^{23}, \mathrm{em} 1980$, têm-se aumentadas as indicaçōes do seu emprego. Exceto para as distonias focais, sua principal indicação, tem sido utilizada, também, no espasmo hemifacial, nos tremores essencial e parkinsoniano, nos tiques, na espasticidade, na acalasia, na hiper-hidrose, na fissura anal e, mesmo, na correção cosmética das rugas ${ }^{3}$. No nosso Serviço, avaliamos os resultados terapêuticos obtidos com o emprego da toxina botulínica do tipo A, em 33 pacientes. Destes, 12 com blefaroespasmo e 11 com torcicolo espasmódico. Nos pacientes com blefaroesapasmo, a dose média empregada ficou em $51,3 \mathrm{U}$, com a duração média do efeito benéfico de 2,8 mcses. Observamos os resultados ótimo em $63,7 \%$; bom, em 22,7\% e nulo, em 13,6\%. Nos pacientes com torcicolo espasmódico, a dose média empregada ficou em $238,6 \mathrm{U}$, com a duração média do efeito benéfico de 4,7 meses. Resultado bom ocorreu em $90 \%$, regular $\mathrm{em} 5 \% \mathrm{c}$ nulo $\mathrm{em} 5 \%$. Concluímos, frente aos nossos resultados ${ }^{18}$, assim como aos da literatura ${ }^{11-16,20,21}$, que a toxina botulínica do tipo A revelou-se eficaz no tratamento destas distonias.

Dentre as drogas de atuação sistêmica, comecemos pelos anticolinérgicos. Em 1979, Fahñ em estudo aberto, observou o benefício obtido nas distonias com o emprego de altas doses de anticolinérgicos. A partir desse estudo, outros foram realizados, duplos cegos e abertos, comprovando os resultados. Todos os estudos mostraram que $50 \%$ das crianças e $40 \%$ dos adultos, com distonia idiopática, obtiveram benefício moderado ou dramático com essa classe de medicamento. Fahn ${ }^{4}$ utilizou o tri-hexifenidil, chegando a $130 \mathrm{mg} / \mathrm{dia}$, aconselhando aumentos gradativos e semanais de $5 \mathrm{mg}$. Obviamente, à medida que as doses vāo sendo aumentadas, os efeitos colaterais periféricos e centrais começam a aparecer. Dentre os periféricos, são mais comuns, boca seca, turvação visual, retenção de urina, constipação intestinal e perda de peso. Dentre os centrais, hipomnésia, confusão mental e alucinaçōes. Para combater a secura na boca, aconselham a co-administração de brometo de piridostigmina; para a turvação visual, gotas de pilocarpina. No que se refere aos efeitos colaterais centrais, somente a redução do tri-hexifenidil. No nosso Serviço, tivemos poucas oportunidades de utilizar este anticolinérgico, como recomendado acima; no entanto, melhores resultados foram observados em crianças, em que a tolerância é maior. De 15 pacientes que utilizaram anticolinérgicos, apenas um obteve excelente resultado, com desaparecimento quase completo dos movimentos anormais; dois, com resultado bom; cinco, regular; quatro, nulo e em três, não houve tempo para avaliação. O tri-hexifenidil atinge o pico sérico em $\pm 1,5$ horas e a sua meia vida é de \pm 4 horas. Estudos a longo prazo (5-15 anos), tanto em criança quanto em adulto, demonstraram que os efeitos colaterais mais sérios, como hipomnésia e distúrbio da atenção, foram reversíveis. Mesmo assim, 
$F_{a h n}{ }^{4}$ aconselha que a dose ideal seja aquela requerida para manter a independência física, isto é, não se deve elevar a dose ao seu limite, na esperança de abolir qualquer evidência de distonia. Observou, ainda, que o único fator indicativo de resposta terapêutica favorável foi o tempo de doença menor que 5 anos; isto é, nāo tinha necessariamente relação com o nível sérico. Quanto ao mecanismo de ação dos anticolinérgicos nas distonias, supõe-se que atue no striatum por ser o locus de importante grupo de neurônios colinérgicos.

Após a introdução de altas doses, por via oral, de levodopa, na doença de Parkinson, esta droga também foi tentada, em pacientes com distonia de torção. Atualmente, sabe-se que somente pequena percentagem de pacientes com distonia pode ter resposta terapêutica. No entanto, pacientes com flutuações diumas de distonia (doença de Segawa) costumam responder dramaticamente às baixas doses de levodopa ou aos agonistas dopaminérgicos ${ }^{7}$. No nosso Serviço, apenas um paciente, com história familiar de distonia, respondeu, favoravelmente, a essa medicação.

É difícil entender porque uma pequena população de pacientes com distonia (10\%) responde favoravelmente à levodopa ou aos agonistas dopaminérgicos, enquanto outra (20\%) responde a drogas de ação oposta (antagonistas dopaminérgicos), isto é, bloqueadores dos receptores da dopamina ou depletoras da dopamina. Uma explicaçāo para esta aparente incongruência é a de que a distonia de torção seja devida a mais de um mecanismo fisiopatológico ${ }^{7}$.

Fenotiazinas, haloperidol, tetrabenazina e pimozide têm sido os antagonistas dopaminérgicos mais utilizados. Marsden e col. (apud Fahn e col. ${ }^{7}$ ), em 1984, sugeriram um coquetel triplice para certos tipos de pacientes graves que não tinham obtido resultados satisfatórios com outras drogas. Desse coquetel constam um depletor de dopamina (tetrabenazina $75 \mathrm{mg} / \mathrm{dia}$ ), um bloqueador do receptor da dopamina (haloperidol ou pimozide) e um anticolinérgico (tri-hexifenidil) por seus efeitos benéficos sobre as distonias, e, também, para prevenir o parkinsonismo induzido por drogas.

Há relatos esparsos na literatura, da ação benéfica de certos farmacos para certos tipos de distonia. Dentre outros, destacam-se o baclofen, o clonazepam, a carbamazepina, o álcool, a clonidina e o carbonato de lítio. Desses, o mais eficaz, desde que seja administrado em aitas doses, é o baclofen ${ }^{7}$. Vários estudos, sobretudo os de Green e Fahn ${ }^{10}$, demonstraram sua eficácia nas distonias, tanto em crianças como em adultos. $O$ baclofen é um derivado do ácido gama-amino butírico (GABA) que inibe o influxo de cálcio e reduz a liberação de transmissores excitatórios como o glutamato $\mathrm{e} o$ aspartato. Empregado na dose média de $80 \mathrm{mg} / \mathrm{dia}$ (40-120 mg), obtiveram melhora importante em $44 \%$ de crianças com distonia. Esta melhora estava relacionada com o tempo de doença (menos de três anos). Na nossa experiência com apenas quatro casos, dois obtiveram resultado regular e dois, nulo.

A abordagem cirúrgica das distonias é conhecida desde a década de 40 . Os procedimentos cirúrgicos podem ser divididos em estereoencefalotomia e lesōes ablativas do sistema nervoso periférico e/ou muscular. A primeira é mais eficaz para as distonias apendiculares, enquanto que as lesōes periféricas, para as focais. Com o aparecimento de novas drogas de atuação sistêmica ou local, a abordagem cirúrgica deve ser recomendada quando não ocorrer resposta satisfatória ao tratamento conservador e quando o movimento anormal for suficientemente grave que impeça as atividades de vida diária.

Gostaríamos de finalizar salientando as experiências pessoais de Marsden e Fahn no que se refere à escolha da primeira droga a ser utilizada. Concluíram que nenhuma droga é uniformemente eficaz, no tratamento das distonias. Desenvolveram, então, uma estratégia terapêutica sintomática que parece razoável na presente circunstância. Dependendo do tipo de distonia, assim aconselham a ordem de escolha dos medicamentos:

a) dentre as distonias focais, no blefaroespasmo, começavam com os anticolinérgicos, seguidos de baclofen e de benzodiazepínicos. Com o melhor conhecimento dos resultados terapêuticos obtidos, 
modernamente, com a toxina botulínica, esta droga é, no momento, a primeira escolha. Na distonia cervical (torcicolo), começam com os anticolinérgicos, e, em seguida, baclofen e carbamazepina, nesta ordem. Se os resultados terapêuticos não forem satisfatórios, empregam a toxina botulínica. Na distonia focal da mão (cāibra profissional), aconselham ao paciente, como primeira medida, a aprender a escrever com a mão homóloga, a despeito de a distonia poder aparecer nessa māo em $30 \%$ dos casos. A droga de escolha nessa condição também é a toxina botulínica.

b) nas distonias segmentares e generalizadas, a levodopa em baixas doses costuma ser a primeira tentativa. Se os resultados não forem favoráveis, empregam os anticolinérgicos, o baclofen ou a carbamazepina.

\section{REFERÊNCIAS}

1. Adams RD, Victor M. Principles of neurology. Ed 5. New York: McGraw-Hill, 1993:56-73.

2. Andrade LAF, Ferraz HB. Idiopathic dystonia: clinical profile of 76 Brazilian patients. Arq Neuropsiquiatr 1992; 50:426-432.

3. Brin MF. Botox Bulletin. International Conference on botulinum toxin: basic science and clinical therapeutics. Munich, Germany, 1995, Vol 1, June 14-16.

4. Fahn S. High dosage anticholinergic therapy in dystonia. Neurology $1983 ; 33: 1255-1261$.

5. Fahn S. Clinical variants of idiopathic torsion dystonia. J Neurol Neurosurg Psychiatry, 1989; Special Suppl:96-100.

6. Fahn S. Psychogenic movements disorders. In Marsden CD, Fahn S (eds). Movement disorders 3. London: Butterworths, 1994: 358-372.

7. Fahn S, Marsden CD, Calne DB. Classification and investigation of dystonia. In Marsden CD, Fahn $\mathbf{S}$ (eds). Movement disorders 2. London: Butterworths, 1987:332-358.

8. Fahn S, Williams DT. Psychogenic dystonia. Adv Neurol 1988; 50:431-455.

9. Ferraz HB, Andrade LAF. Symptomatic dystonia: clinical profile on 46 Brazilian patients. Can J Neurol Sci 1992; 19:504-507.

10. Greene P, Fahn S. Patterns of spread of dystonia in childhood onset dystonia. Neurology 1987; 37(Suppl 1):269.

11. Greene $P$, Fahn $S$. Use of botulinum toxin type $F$ injections to treat torticollis in patients with immunity to botulinum toxin type A. Mov Disord 1993; 8:479-483.

12. Greene P. Fahn S, Brin MF, Blitzer A. Botulinum toxin therapy. In Marsden CD, Fahn S (eds). Movement Disorders 3. London: Butterworths, 1994: 477-502.

13. Greene P, Fahn S, Diamond B. Development of resistance to botulinum toxin type A in patients with torticollis. Mov Disord 1994; 9:213-217.

14. Jankovic J, Brin MF. Therapeutic uses of botulinum toxin. N Engl .J Med 1991; 324:1186-1194.

15. Lees AJ. Botulinum toxin: useful in adult onset focal dystonias. BMJ 1992; 305: $1169-1170$

16. Lorentz IT, Subramaniam SS, Yiannikas C. Treatment of idiopathic spasmodic torticollis with botulinum toxin A: a double-blind study on twenty-three patients. Mov Disord 1991; 6:145-150.

17. Marsden CD, Harrison MJG, Bundey S. Natural history of idiopathic torsion dystonia Adv Neurol 1976; 14:177-186.

18. Novis S, Mattos JP, Rosso ALZ. Toxina botulínica no blefaroespasmo, no espasmo hemifacial e na distonia cervical: resultados em 33 pacientes. Arq Neuropsiquiatr 1995; 53:403-410.

19. Oppenheim H. Uber eine eigenartige Krampfkrankheit des Kindlichen und Jungendlichen Alters (Dysbasia lordotica progressiva, Dystonia musculorum deformans). Neurol Centralbl 1911b; 30:1090-1107.

20. Savino PJ, Maus M. Botulinum toxin therapy. Neurol Clin 1991; 9:205-224.

21. Schneider S, Heinen F, Feitel E, Köster B, Maximov M, Deuschel G. Longterm results of botulinum toxin treatment. Mov Disord 1994; 9:47.

22. Schwalbe W. Eine eigentumliche tonische Krampfform mit hysterischen Symptomen. Thesis (Inaug.Diss). Berlin: G.Schade, 1908.

23. Scott AB. Botulinum toxin injection into extraocular muscles as an alternative to strabismus surgery.Ophthalmology 1980; 87:1044-1049. 